\title{
An Unusual Case of Contact Dermatitis
}

\author{
Olivia B. Hughes Andrea D. Maderal Antonella Tosti
}

Department of Dermatology and Cutaneous Surgery, University of Miami Miller School of Medicine, Miami, FL, USA

\section{Established Facts}

- Low-level light therapy (LLLT) is increasingly popular in the treatment of hair loss with numerous devices on the market including combs, hats, hoods, and helmets. Although the mechanism of action of LLLT is unknown, it has been hypothesized that light may activate dormant hair follicles, increase blood flow, and upregulate the production of growth factors and adenosine triphosphate in order to stimulate anagen hair.

\section{Novel Insights}

- This is the first case of allergic contact dermatitis caused by a low-level light therapy cap.

\section{Keywords}

Low-level light therapy · Psoriasiform contact dermatitis . Dimethylol dihydroxyethyleneurea · Allergy $\cdot$ Hair · Hair loss

\begin{abstract}
We report a case of acute psoriasiform contact dermatitis of the forehead and scalp related to the use of a low-laser light cap. The patient had a positive patch test to dimethylol dihydroxyethyleneurea (DMDE), methylisothiazolinone, kathon CG, and cobalt. We believe that DMDE in the fabric of the cap was responsible for the allergic contact dermatitis in this case as the lesions resolved with cessation of cap use.
\end{abstract}

(c) 2017 S. Karger AG, Basel

\section{Introduction}

Low-level light therapy (LLLT) is quite popular in the treatment of androgenetic alopecia (AGA) with different types of devices such as combs, hoods, caps, or helmets.
LLLT devices are considered safe, and contact allergy to them has not been reported in the literature. In a multicenter, randomized, sham device-controlled, double blind study of the safety and efficacy of low-level laser therapy in the treatment of AGA, there were no serious adverse events reported in any of the 269 subjects. Dry skin $(5.1 \%)$, pruritus $(2.5 \%)$, scalp tenderness $(1.3 \%)$, irritation $(1.3 \%)$, and a warm sensation at the site $(1.3 \%)$ were reported [1].

\section{Case Report}

A 65-year-old male was referred to the clinic with a 2-year history of scalp dermatitis and itching. The patient has AGA, for which he has undergone multiple hair transplants in the past. His current treatments included 5\% minoxidil solution (a private dermatologist's brand), which he has been using for the past 5 years and a low-level laser cap that he has been wearing daily for $30 \mathrm{~min}$. The laser cap is lined with plastic and rubber and contains an interior metal probe. It is designed to be inserted inside a hat; therefore, the patient alternated between wearing the cap from the LLLT company and a baseball cap to cover it and keep it in place.

\section{KARGER}

() 2017 S. Karger AG, Basel 

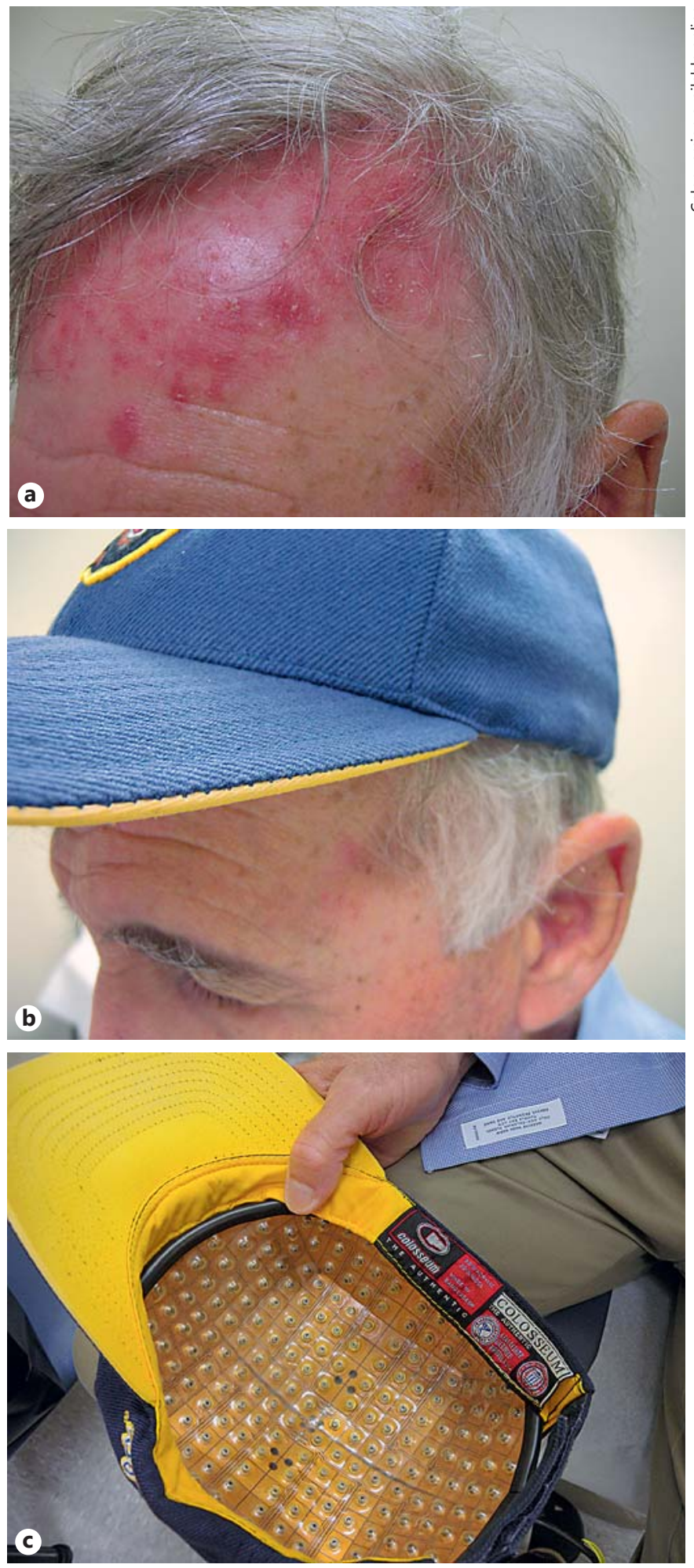

Fig. 1. a The patient has sharply demarcated, erythematous, scaly plaques with overlying excoriations on the scalp extending to the frontal hairline, corresponding to the area in contact with the cap. b The cap distribution is consistent with the allergic contact dermatitis distribution. c The low-level light therapy cap device.

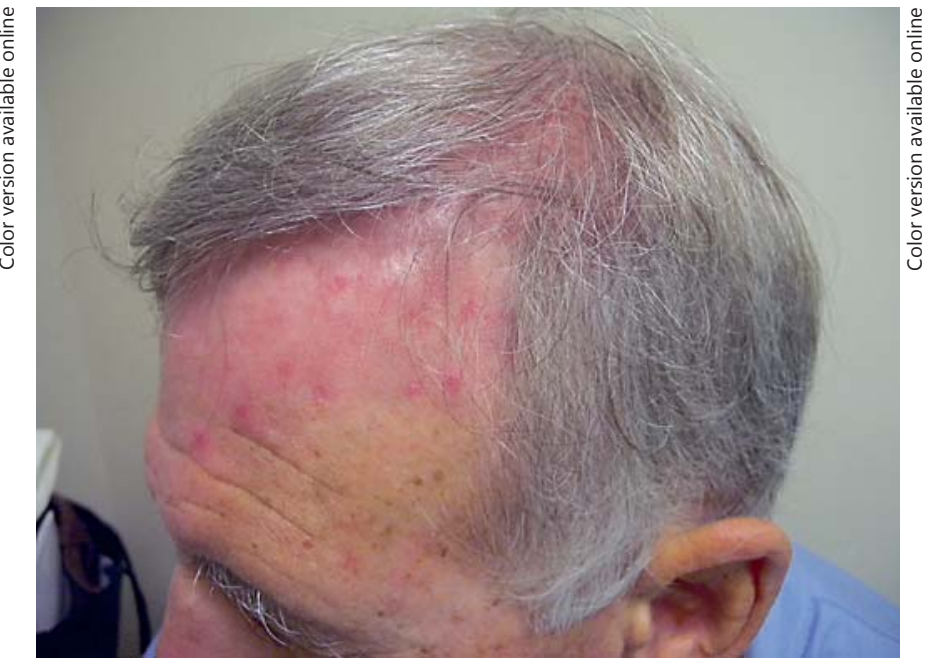

Fig. 2. Resolution of the rash after avoidance of the cap.

On physical examination, the patient had sharply demarcated, erythematous, scaly plaques with overlying excoriations on the scalp extending to the frontal hairline, corresponding to the area in contact with the cap (Fig. 1a-c). It had the appearance of classic psoriatic plaques; however, the presence of pruritus and the distribution consistent with the cap caused us to consider ACD. Seborrheic dermatitis was excluded from the list of differential diagnoses because the patient's lesions lacked the characteristic greasy-looking yellow scale. Dermoscopic examination of the scalp revealed arborizing vessels. The patient had 3 biopsies that were all consistent with contact dermatitis. Despite psoriasis being on the list of differential diagnoses, features of psoriasis were not observed in any biopsy.

Patch testing with the North American standard series of 50 allergens revealed 2+ reactions to kathon CG, methylisothiazolinone, cobalt, and dimethylol dihydroxyethyleneurea (DMDE). A cobalt spot test on the metal laser cap was negative. The patient was instructed to use a shampoo without isothiazolinones and to stop wearing the laser device. Further patch tests with the cosmetic series of 48 allergens, textile series of 39 allergens, fragrance series of 41 allergens, the patient's sunscreen, the private dermatologist's brand of topical minoxidil solution, and the minoxidil vehicle were all negative. Treatment with topical steroids and discontinuation of the laser cap and baseball hat considerably improved the skin lesions (Fig. 2).

\section{Discussion}

This patient had an unusual psoriatic-like presentation of a severe contact dermatitis of the scalp associated with the use of a low-level laser device and baseball cap. Unfortunately, we were not able to define with certitude which allergen was causing the dermatitis in this patient. However, DMDE in the cap is most likely the agent that 
caused the allergic skin reaction as there was fast improvement of the skin lesions after LLLT cap and baseball cap avoidance and return of the rash when wearing either of the caps (which were not $100 \%$ cotton) despite cessation of all other therapies and cosmetics including minoxidil. Although the manufacturer of the device assured that its internal surface did not contain metals, and the cobalt spot test of the cap was negative, we cannot completely exclude the role of cobalt as the spot test may cause false-negative results and the manufacturer may not be completely aware of its composition. The patient's shampoo did not contain isothiazolinones, and he denied using wipes or other products that could potentially contain the preservative to clean the surface of the device.

DMDE is a formaldehyde-releasing agent that enhances the self-cleaning performance, UV-blocking, and antibacterial properties of some fabrics [2]. DMDE is likely present in the LLLT cap and baseball cap utilized to keep the device in place. The LLLT cap was made in Bangladesh, and its label declared that it was composed of $20 \%$ wool and $80 \%$ acrylic, and the baseball cap was not $100 \%$ cotton. A diagnosis of ACD to resins such as DMDE in permanent-press textiles is made based upon the clinical finding of a rash in the distribution of the item of clothing, possibly with sparing where undergarments are pres- ent, as well as a positive patch test $[3,4]$. Various clinical reaction patterns have been reported to DMDE, including a purpuric ACD as well as the classic erythematous patch or plaque with vesicles, blisters, and/or scale $[2,4$, 5]. There have been case reports of ACD to formaldehyde and formaldehyde resins in nail hardeners that mimic psoriasis; however, this is the first case to be reported of a psoriatic-like ACD to DMDE [6]. In our case, the patient had positive patch test results to DMDE and had dermatitis in the distribution of his baseball hat and laser cap. In the overall context of a suggestive clinical history, appropriate distribution, positive patch test findings, and improvement with discontinuation of the use of the laser cap, the diagnosis of ACD to DMDE from the laser cap and baseball hat was confirmed.

\section{Statement of Ethics}

The patient has given informed consent to allow this case to be published.

\section{Disclosure Statement}

The authors do not have any conflicts of interest to disclose.
References

An Unusual Case of Contact Dermatitis
1 Jimenez JJ, Wikramanayake TC, Bergfeld W, Hordinsky M, Hickman JG, Hamblin MR, Schachner LA: Efficacy and safety of a lowlevel laser device in the treatment of male and female pattern hair loss: a multicenter, randomized, sham device-controlled, doubleblind study. Am J Clin Dermatol 2014;15: 115-127.

2 Scheman AJ, Carroll PA, Brown KH, Osburn $\mathrm{AH}$ : Formaldehyde-related textile allergy: an update. Contact Dermatitis 1998;38:332-336.

3 Metzler-Brenckle L, Rietschel RL: Patch testing for permanent-press allergic contact dermatitis. Contact Dermatitis 2002;46:33-37.
4 Bolognia J, Jorizzo JL, Schaffer JV: Dermatology (ed 3). Philadelphia, Elsevier Saunders, 2012, vol 1, pp 209-240.

5 Lazarov A, Cordoba M: Purpuric contact dermatitis in patients with allergic reaction to textile dyes and resins. J Eur Acad Dermatol Venerol 2000;14:101-105.

6 Mestach L, Goossens A: Allergic contact dermatitis and nail damage mimicking psoriasis caused by nail hardeners. Contact Dermatitis 2015;74:112-114. 